\title{
In vitro effectiveness of Anidulafungin against Candida sp. biofilms
}

\author{
Antonio Rosato ${ }^{1}$, Monica Piarulli ${ }^{2}$, Brigida Pia Immacolata Schiavone ${ }^{2}$, Alessia Catalano ${ }^{1}$, Alessia Carocci ${ }^{1}$, \\ Antonio Carrieri ${ }^{1}$, Addolorata Carone ${ }^{1}$, Giuseppina Caggiano ${ }^{3}$, Carlo Franchini ${ }^{1}$, Filomena Corbo ${ }^{1}$ \\ and Maria Teresa Montagna ${ }^{3}$
}

This study furnishes deeper insights to previous works on anidulafungin, demonstrating the potent activity against Candida strains planktonic cells and biofilms. Candida sp., associated with many biomaterial-related infections, give rise to infective pathologies typically associated with biofilm formation. We recently determined the in vitro antifungal activities of echinocandin anidulafungin in association with some antifungal drugs against some Candida strains in their planktonic states. A total of 11 Candida strains biofilms were tested in this study: six Candida albicans, three $C$. parapsilosis and two $C$. tropicalis. All yeast isolates and ATCC strains were stored at $-20^{\circ} \mathrm{C}$ in glycerol stocks and were subcultured on antimicrobial agent-free Sabouraud dextrose agar plates. MIC endpoints were determined colorimetrically by using the indicator 2,3-bis(2-methoxy-4-nitro-5sulphophenyl)-5-[(phenylamino)carbonyl]-2 $\mathrm{H}$-tetrazolium hydroxide (XTT) with menadione as electron-coupling agent. The activity of anidulafungin was assessed using in vitro microbiological model relevant for clinical practice. Anidulafungin showed a strong activity in vitro against both planktonic and biofilms cells, and our study confirms that high anidulafungin concentrations might establish paradoxical growth effect in $\boldsymbol{C}$. albicans and $C$. tropicalis biofilms. The Journal of Antibiotics (2013) 66, 701-704; doi:10.1038/ja.2013.83; published online 11 September 2013

Keywords: anidulafungin; antifungal agent; biofilm; Candida sp.; paradoxical effect; XTT

\section{INTRODUCTION}

Some pathogenic species in the Candida genus are known to seriously cause both superficial and systemic infections now widely recognized in modern clinical practice. ${ }^{1}$

Most Candida infections involve biofilm formation on implanted devices (indwelling catheters) and tissue surfaces, being the adhesion of the yeast to host surface or associated prosthesis, such as a denture or intravascular catheter material, the first step of the same infection. ${ }^{2}$ During the last period the capacity of Candida sp. to form biofilms has been evaluated, and special attention has been focused on the frequent use of clinical devices. ${ }^{3-5}$ In fact, the failure of antifungal therapy lead to chronic infections that might be recovered only by surgery and/or removal of these medical devices. Therefore, an antifungal lock therapy with a high concentration of antibiotics could avoid systemic toxicity.

Lower antifungal activities have been commonly proved for amphotericin B, fluconazole and itraconazole, and these antifungal agents are indeed much less active against Candida sp. biofilms. However, a new class of antifungal agents, namely echinocandins (ECs) and in particular anidulafungin (ANF), active in vitro against Candida sp. has been recently introduced; it has been recently demonstrated that ECs might be active against Candida sp. biofilmassociated infections frequently refractory to conventional therapy ${ }^{6-8}$ being able to inhibit the synthesis of $\beta$-1,3-glucan, an important component of the structure of fungal cell wall ${ }^{9-11}$ as proved by important in vitro and in vivo antifungal activity. More than few studies have been carried out mainly on the use of caspofungin as $\mathrm{EC}^{7-10}$ acting on C. albicans and non albicans Candida biofilms; ${ }^{11-14}$ recently a study of ANF and micafungin was also carried out on Candida tropicalis biofilm. ${ }^{15}$ Finally, a comprehensive report comparing the in vitro activities of three ECs (ANF, caspofungin and micafungin) against biofilm formed by different non Candida sp. has been recently published. ${ }^{16}$ In this latter paper, ANF results among the three antifungal drugs taken into account as the most interesting EC. In the majority of the cited papers, a growth in the presence of EC concentrations above the MIC in broth microdilution susceptibility test, performed according NCCLS guidelines, has been described. This phenomenon was named paradoxical growth effect (PGE) and appears to be species-strain and EC specific. ${ }^{15,17,18}$ To date, the clinical significance of the PGE remains unclear. Moreover, as antifungal lock therapy with high concentrations of antibiotic represents a good practise for medical devise candidemia, it is

${ }^{1}$ Dipartimento di Farmacia-Scienze del Farmaco, Università degli Studi di Bari 'Aldo Moro', Via Orabona 4, Bari Italy; ${ }^{2}$ Scuola di Dottorato e Scienze Biomediche e Oncologia umana, Sezione di Igiene, Università di Bari 'Aldo Moro', Bari, Italy and ${ }^{3}$ Dipartimento di Scienze Biomediche e Oncologia umana, Sezione di Igiene, Università di Bari 'Aldo Moro', Bari, Italy

Correspondence: Professor A Rosato, Department of Pharmacy, Università degli Studi di Bari, via Orabona 4, 70125, Bari, Italy.

E-mail: arosato@farmchim.uniba.it

Received 5 March 2013; revised 28 May 2013; accepted 30 July 2013; published online 11 September 2013 
important to understand the phenomena of biofilm PGE in the presence of high EC concentrations. In this scenario, in this paper, we focused our attention in particular on in vitro activity of ANF against four Candida ATCC strains and seven Candida isolates growing in planktonic and sessile states in order to understand the contribution of Candida biofilm PGE in vivo and the potential application of ANF to lock therapy.

\section{MATERIALS AND METHODS Organisms}

A total of 11 yeast strains were used in this study: four collection strains (C. albicans ATCC 10231, C. albicans ATCC 90028, C. parapsilosis ATCC 22019, C. tropicalis ATCC 750) and seven isolate strains (C. albicans BA 10A12, C. albicans BA 18, C. albicans BA 17A18, C. albicans BA 18A19, C. parapsilosis BA 11A13, C. parapsilosis BA 1A, C. tropicalis BA 12). Seven isolates of C. albicans and non albicans Candida sp. were recovered from adult immunocompromised and critically ill patients. The strains were isolated from different clinical specimens and derived from blood cultures including C. albicans BA 17A18 from patient with intravascular catheter. All the isolates were from patients admitted to the intensive care unit of Department of Biomedical Science and Human Oncology of Bari, Italy and were recovered between January 2009 and January 2010. They were identified by sugar assimilation profiles, using the biochemical tests performed with the commercial system API ID32C (BioMerieux, Marcy l'Etoile, France). Stocks were maintained at $-80{ }^{\circ} \mathrm{C}$ in yeast peptone dextrose broth with 10-25\% glycerol (Oxoid, Milano, Italy) solution. All isolates yeast were stored at $-20{ }^{\circ} \mathrm{C}$ in glycerol stocks and before the initiation of the study, they were subcultured on antimicrobial agent-free Sabouraud dextrose agar plates (BioMerieux, Marcy l'Etoile, France) to ensure viability and purity. C. albicans ATCC 10231, ATCC 90028, C. parapsilosis ATCC 22019 and C. tropicalis ATCC 750 were used as quality controls and tested in all experiments.

\section{Medium and culture conditions}

Each frozen stock culture, maintained at $-20^{\circ} \mathrm{C}$ was inoculated onto Sabouraud dextrose broth (Difco, Milano, Italy) and incubated at $37^{\circ} \mathrm{C}$ for $24 \mathrm{~h}$ in an orbital shaker at 60 r.p.m. Cells were picked and added to a tube containing RPMI 1640 broth medium with L-glutamine and without bicarbonate buffered to $\mathrm{pH} 7$ with MOPS (3-( $\mathrm{N}$-morpholino) propanesulfonic acid (165 M, Sigma, Milano, Italy). Consequently, a standardized suspension of $1 \times 10^{6} \mathrm{CFU} \mathrm{ml}{ }^{-1}$ was obtained and immediately used.

\section{Antifungal agent and material}

ANF was supplied by Pfizer (Rome, Italy). Flat circular polyvinyl chloride disks measuring $13 \mathrm{~mm}$ in diameter and $2 \mathrm{~mm}$ in thickness were obtained from Mediatipo (Bari, Italy). The disks were washed in laboratory detergent, rinsed three times in distilled water and ethanol 30\%, air dried and sterilized in autoclave before use.

\section{Planktonic cells MICs.}

The susceptibility of planktonic cells was determined by the 2,3-bis(2methoxy-4-nitro-5-sulphophenyl)-5-[(phenylamino)carbonyl]-2H-tetrazolium hydroxide (XTT) reduction assay.

The MIC obtained by the use of XTT was defined as the lowest drug concentration that caused a $50 \%$ decrease in metabolic activity relative to that in the drug-free growth control. ${ }^{13}$

\section{Biofilm formation and testing}

The sessile MIC (SMIC) values were determined using XTT cell viability assay Autoclaved disks, containing $2 \mathrm{ml}$ of $1 \times 10^{6} \mathrm{CFU} \mathrm{ml}^{-1}$ Candida cells suspension, were placed one disk per well in 24-well tissue culture plates (Corning, Corning, Milan, Italy). The plates were incubated at $37^{\circ} \mathrm{C}$ for $1.5 \mathrm{~h}$ with shaking at 150 r.p.m. on a rocker table enabling the Candida cells attachment.

After the $45 \mathrm{~h}$ maturation period, the media was removed and the biofilms gently rinsed with sterile PBS, and the disks were transferred to another 24-well plate containing the antifungal agent.

Biofilm growth was quantified colorimetrically by a solution of XTT with menadione as the electron-coupling agent.

Plates were incubated at $37^{\circ}$ for $2 \mathrm{~h}$, and the medium was removed and centrifuged for $30 \mathrm{~min}$ at $3000 \mathrm{~g}$ to pellet any suspended cells or debris. The amount of XTT-formazan in the supernatant was measured at $490 \mathrm{~nm}$ by using a spectrophotometer. For susceptibility testing (SMIC), biofilms were formed in RPMI 1640 medium. After $45 \mathrm{~h}$, preformed biofilm-containing disks were washed with sterile PBS prior to test with ANF diluted in RPMI 1640 medium, to yield 9 doubling serial dilutions ranging from 0.25 to $128 \mu \mathrm{g} \mathrm{ml}^{-1}$. The disks were transferred to new culture plates containing RPMI 1640 medium $(2 \mathrm{ml})$ and different concentrations of the antifungal agents. After exposure to the antifungal agents for $24 \mathrm{~h}$ at $37^{\circ} \mathrm{C}$ on a rocker table, biofilm activity was measured by XTT reduction assay as described above. The antifungal concentration causing $50 \%$ reduction of the biofilm compared with the metabolic activity of the drug-free control was then determined (SMICs). Antimicrobial susceptibility tests of biofilms were performed in triplicate.

Table 1 Antifungal susceptibilities and paradoxical effect of different Candida spp. strains under planktonic and biofilm growth conditions as determined using the $X T T$ method $^{\mathrm{a}}$

\begin{tabular}{|c|c|c|c|}
\hline \multirow[b]{2}{*}{ Strains } & \multicolumn{2}{|c|}{ MIC $\left(\mu g \mathrm{ml}^{-1}\right)$ of anidulafungin for } & \multirow[b]{2}{*}{ Paradoxical effec } \\
\hline & Planktonically grown cells ${ }^{\mathrm{b}}$ & Biofilms at $24 h(S M I C 50)^{b}$ & \\
\hline C. albicans ATCC 10231 & 0.01 & 0.5 & 4.0 \\
\hline C. albicans ATCC 90028 & 0.01 & 0.5 & 4.0 \\
\hline C. albicans BA $10 \mathrm{~A} 12$ & 0.12 & 0.5 & 1.0 \\
\hline C. albicans BA 18 & 0.12 & 0.5 & 2.0 \\
\hline C. albicans BA $17 \mathrm{~A} 18$ & 0.12 & 0.5 & 2.0 \\
\hline C. albicans BA 18 A 19 & 0.12 & 0.5 & 2.0 \\
\hline C. parapsilosis ATCC 22019 & 1.0 & 32.0 & c \\
\hline C. parapsilosis BA $11 \mathrm{~A} 13$ & 0.5 & 16.0 & c \\
\hline C. parapsilosis $\mathrm{BA} 1 \mathrm{~A} 1$ & 0.5 & 16.0 & c \\
\hline C. tropicalis ATCC 750 & 0.01 & 1.0 & 8.0 \\
\hline C. tropicalis BA 12 & 0.12 & 0.5 & 4.0 \\
\hline
\end{tabular}

Abbreviation: SMIC, sessile MIC

aResults are representative of three separate experiments.

bThe MIC end point for planktonic cells and biofilms is based on the lowest drug concentration producing a decrease of $50 \%$ metabolic activity relative to metabolic activity of the untreated growth control (XTT reduction assay).

'These strains did not show any paradoxical effect. 


\section{RESULTS}

The susceptibility of planktonic cells and biofilms to ANF are shown in Table 1. The planktonic cells were highly susceptible to the ANF except for C. parapsilosis strains tested, which was less susceptible than the other species. In fact, the MICs ranged between 0.01 and $0.12 \mu \mathrm{g} \mathrm{ml}^{-1}$ for C. albicans and C. tropicalis strains, whereas ranged between 0.5 and $1 \mu \mathrm{g} \mathrm{ml}^{-1}$ for C. parapsilosis. As shown, the sessile cells MICs (SMICs) of ANF, indicating the 50\% reductions in terms of metabolic activity, were not comparable to the planktonic cell MICs for all our fungal strains. The SMICs for biofilms of C. albicans ATCC 10231, ATCC 90028, C. tropicalis ATCC 750 and C. albicans and C. tropicalis isolates ranged between 0.5 and $1 \mu \mathrm{g} \mathrm{ml}^{-1}$, more than 5 or 6 dilutions higher than their planktonic MICs (Table 1). The SMICs for all C. parapsilosis strains were six dilutions higher than the planktonic MICs. Despite the growth inhibitory activity of ANF at low concentrations, a paradoxical growth at supra MICs, followed by growth inhibition in the presence of the highest drug concentrations has been observed for some Candida sp. ATCC and isolates. In fact the susceptibility pattern exhibited by C. tropicalis ATCC 750 C. tropicalis BA12, C. albicans ATCC 10231 and C. albicans BA10a12, in which biofilm cells were less susceptible to ANF at concentrations above the SMIC $\left(0.5-16 \mu \mathrm{g} \mathrm{ml}^{-1}\right)$, might be intended as a PGE. ${ }^{15}$ Vice versa the same effect for ANF was not observed in C. parapsilosis strains; overall, these results related to our experimental study suggest that ANF is more effective against preformed C. albicans and C. tropicalis biofilms with respect to C. parapsilosis biofilms. These results are in agreement with a previous paper in which biofilm of C. parapsilosis was more refractory to ANF than with the other antifungal agents. ${ }^{16,19}$

\section{DISCUSSION}

As reported by several research works, ${ }^{20-23}$ Candida sp. biofilms show strong resistance to a wide spectrum of conventional antifungal agents as azoles; in fact Candida biofilms are reported as resistant to the new triazoles (voriconazole and ravuconazole), which have shown an extended spectrum of activity against many azole-resistant organisms as well as evidence of fungicidal, rather than fungistatic, activity. Two novel classes of agents, the lipid formulation amphotericins and the ECs, seem to have unique activities against these Candida biofilms. Our study confirms this result for our isolates, showing ANF's strong inhibitory action against Candida biofilms. Another aspect of our research has been the evaluation of PGE. Our study highlights the PGE confirming several previous research reports. In fact despite the growth inhibitory activity of ANF at low concentrations, paradoxical growth at supra MICs followed by growth inhibition in the presence of the highest ANF concentration has been observed for our C. albicans and C. tropicalis strains. Several explanations have been presented about the possible correlation of PGE and high concentration of ECs drugs. ${ }^{16,19,24,25}$ However, as previously reported, ANF belonging to the ECs family is able to inhibit the synthesis of $\beta$-1,3-glucan, a fundamental component of fungal cell wall, by inhibition of $\beta$-1,3-glucan synthase. ${ }^{22}$ Stevens et al. ${ }^{30}$ proposed a plausible justification of the PGE measuring the level of $\beta$-1,3-glucan, $\beta$-1,6-glucan and chitin in a $C$. albicans strains after exposure to high caspofungin. ${ }^{26}$

Regardless, a decrease of both the $\beta$-1,3-glucan and the $\beta-1,6$ glucan content with respect to the control (untreated cells), chitin concentrations increased significantly after drug exposure. This might suggest that cell wall composition is affected by caspofungin exposure, leading then to a modification of cell morphology. ${ }^{27}$ Other mechanisms explaining the paradoxical effect include involvement of the calcineurin pathway and upregulation of the protein kinase- $\mathrm{C}$ cell wall integrity pathway. ${ }^{23}$ From experimental point of view and as previously reported, we applied the in vitro use of $\mathrm{XTT}^{28}$ in the determination of ANF activity against ATCC and isolates fungal strain biofilms. $^{28,29}$ The procedure simplifies biofilm formation and quantification, making it more reliable and comparable among different laboratories, a necessary step toward the standardization of antifungal susceptibility testing of biofilms. As previously published by others, ${ }^{5,13,15}$ we assumed that measurements based on XTT metabolic activity were sufficient to indirectly quantify biofilms. In conclusion, till date there are no studies about the effect of ANF on non albicans Candida sp. except for a recent study on C. tropicalis ATCC strains ${ }^{15}$ and the comparative study on less common bloodstream Candida isolates. ${ }^{16}$ Therefore, the results related to our isolates and ATCC strains, reported in this paper, demonstrated that in vitro therapeutic concentrations of ANF display potent activity against C. albicans and C. tropicalis biofilm and planktonic cells with an important paradoxical growth. Moreover, lower SMICs were recorded in this research for C. parapsilosis ATCC and isolates with no PGE, according to the results of Melo; ${ }^{15}$ therefore, our study confirmed again that $C$. parapsilosis biofilm are substantially more resistant to ANF than other Candida sp. Although, the clinical significance of $\mathrm{PGE}^{30}$ is not clear, this could theoretically have an impact on the potential use of ANF in the lock therapy for the management of infected intravascular catheters. Nonetheless, further work involving (also based on previous data ${ }^{31,32}$ ) in vitro and in vivo experiments is needed in order to determine the validity of our observations.

\section{CONFLICT OF INTEREST}

The authors declare no conflict of interest.

\section{ACKNOWLEDGEMENTS}

This study was supported by an unrestricted grant from Pfizer s.r.l. Italy.

1 Cox, G. M. \& Perfect, J. R. Fungal infections. Curr. Opin. Infect. Dis. 6, 422-426 (1993).

2 Koijc, E. M. \& Darouiche, R. O. Candida infections of medical devices. Clin. Microbiol. Rev. 17, 255-267 (2004).

3 Chandra, J. et al. Biofilm formation by the fungal pathogen Candida albicans: development, architecture, and drug resistance. J. Bacteriol. 183, 5385-5394 (2001).

4 Douglas, L. J. Candida biofilms and their role in infection. Trends Microbiol. 11, 30-36 (2003).

5 Hawser, S. P., Norris, H., Jessup, C. J. \& Ghannoum, M. A. Comparison of a 2,3-bis(2methoxy-4-nitro-5-sulfophenyl)-5-[(phenylamino)carbonyl]- $2 \mathrm{H}$-tetrazolium hydroxide (XTT) colorimetric method with the standardized National Committee for Clinical Laboratory Standards method of testing clinical yeast isolates for susceptibility to antifungal agents. J. Clin. Microbiol. 36, 1450-1452 (1998).

6 Kuhn, D. M. \& Ghannoum, M. A. Candida biofilms: antifungal resistance and emerging therapeutic options. Curr. Opin. Invest. Dr. 5, 186-197 (2004).

7 Kuhn, D. M., George, T., Chandra, J., Mukherjee, P. K. \& Ghannoum, M. A. Antifungal susceptibility of Candida biofilms: unique efficacy of amphotericin B lipid formulations and echinocandins. Antimicrob. Agents Chemother. 46, 1773-1780 (2002).

$8 \mathrm{Kim}$, R., Khachikia, D. \& Reboli, A. C. A comparative evaluation of properties and clinical efficacy of the echinocandins. Expert Opin. Pharmacother. 8, 1479-1492 (2007).

9 Bachmann, J. S. P. et al. In vitro activity of CSP against Candida albicans biofilms. Antimicrob. Agents Chemother. 46, 3591-3596 (2002).

$10 \mathrm{Choi}, \mathrm{H}$. W. et al. Species-specific differences in the susceptibilities of biofilms formed by Candida bloodstream isolates to ECs. Antifungals. Antimicrob. Agents Chemother. 51, 1520-1523 (2007).

11 Jacobson, M. J., Piper, K. E., Nguyen, G., Steckelberg, J. M. \& Patel, R. In vitro activity of anidulafungin against Candida albicans biofilms. Antimicrob. Agents Chemother. 52, 2242-2243 (2008). 
12 Cocuaud, C., Rodier, M. H., Daniault, G. \& Imbert, C. Anti-metabolic activity of CSP against Candida albicans and Candida parapsilosis biofilms. J. Antimicrob. Chemother. 56, 507-512 (2005)

13 Ferreira, J. A. G., Carr, J. H., Starling, C. E. F., De Resende, M. A. \& Donlan, R. M. Biofilm formation and effect of CSP on biofilm structure of Candida species bloodstream isolates. Antimicrob. Agents Chemother. 53, 4377-4384 (2009).

14 Miceli, M. H., Bernardo, S. M. \& Lee, S. A. In vitro analyses of the combination of highdose doxycycline and antifungal agents against Candida albicans biofilms. Int. J. Antimicrob. Agents 34, 326-332 (2009).

15 Melo, A. S., Colombo, A. L. \& Arthington-Skaggs, B. A. Paradoxical growth effect of CSP observed on biofilms and planktonic cells of five different Candida species. Antimicrob. Agents Chemother. 51, 3081-3088 (2007).

16 Simitsopoulou, M. et al. Species-specific and drug-specific differences in susceptibility of Candida biofilms to echinocandins: characterization of less common bloodstream isolates. Antimicrob. Agents Chemother. 57, 2562-2570 (2013).

17 Chamilos, G., Lewis, R. E., Albert, N. \& Kontoyiannis, D. P. Paradoxical effect of ECs across Candida species in vitro: evidence for echinocandin-specific and Candida species-related differences. Antimicrob. Agents Chemother. 51, 2257-2259 (2007).

18 Fleischhacker, M., Radecke, C., Schulz, B. \& Ruhnke, M. Paradoxical growth effects of the ECs CSP and micafungin, but not of anidulafungin, on clinical isolates of Candida albicans and C. dubliniensis. Eur. J. Clin. Microbiol. Infect. Dis. 27, 127-131 (2008).

19 Wiederhold, N. P. Attenuation of echinocandin activity at elevated concentrations: a review of the paradoxical effect. Curr. Opin. Infect. Dis 20, 574-578 (2007).

20 Mukherjee, P. K. \& Chandra, J. Candida biofilm resistance. Drug Resist Update 7, 301-309 (2004).

21 Hawser, S. P. \& Douglas, L. J. Resistance of Candida albicans biofilms to antifungal agents in vitro. Antimicrob. Agents Chemother 39, 2128-2131 (1995).

22 Mishra, N. N. et al. Pathogenicity and drug resistance in Candida albicans and other yeast species. A review. Acta Microbiol. Immunol. Hung. 54, 201-235 (2007).
23 d'Enfert, C. Biofilms and their role in the resistance of pathogenic Candida to antifungal agents. Curr. Drug Targets 7, 465-470 (2006).

24 Ramage, G., Wickes, B. L. \& Lopez-Ribot, J. L. Biofilms of Candida albicans and their associated resistance to antifungal agents. Am. Clin. Lab. 20, 42-44 (2001).

25 LaFleur, M. C., Kumamoto, C. A. \& Lewis, K. Candida albicans biofilms produce antifungal-tolerant persister cells. Antimicrob. Agents Chemother. 50, 3839-3846 (2006).

26 Georgopapadakou, N. H. Update on antifungals targeted to the cell wall: focus on $\beta-1,3$-glucan synthase inhibitors. Expert Opin. Investig. Drugs 10, 269-280 (2001).

27 Alem, M. A. \& Douglas, L. J. Effects of aspirin and other non steroidal antiinflammatory drugs on biofilms and planktonic cells of Candida albicans. Antimicrob. Agents Chemother. 48, 41-47 (2004).

28 Kuhn, D. M., Balkis, M., Chandra, J., Mukherjee, P. K. \& Ghannoum, M. A. Uses and limitations of the XTT assays in studies of Candida growth and metabolism. J. Clin. Microbiol. 41, 506-508 (2003).

29 Kuhn, D. M., George, T., Chandra, J., Mukherjee, P. K. \& Ghannoum, M. A Antifungal susceptibility of Candida biofilms: unique efficacy of amphotericin B lipid formulations and echinocandins. Antimicrob. Agents Chemother. 46, 1773-1780 (2002).

30 Stevens, D. A., Ichinomiya, M., Koshi, Y. \& Horiuchi, H. Escape of Candida from CSP inhibition at concentrations above the MIC (paradoxical effect) accomplished by increased cell wall chitin: evidence for -1,6-glucan synthesis inhibition by CSP. Antimicrob. Agents Chemother. 50, 3160-3161 (2006).

31 Rosato, A. et al. In vitro synergy testing of anidulafungin with fluconazole, tioconazole, 5-flucytosine and amphotericin B against some. Candida sp. Med. Chem 8, 690-698 (2012).

$32 \mathrm{Ku}$, T. S., Bernardo, S. M. \& Lee, S. A. In vitro assessment of the antifungal and paradoxical activity of different echinocandins against Candida tropicalis biofilms. J. Med. Microbiol. 60, 1708-1710 (2011). 